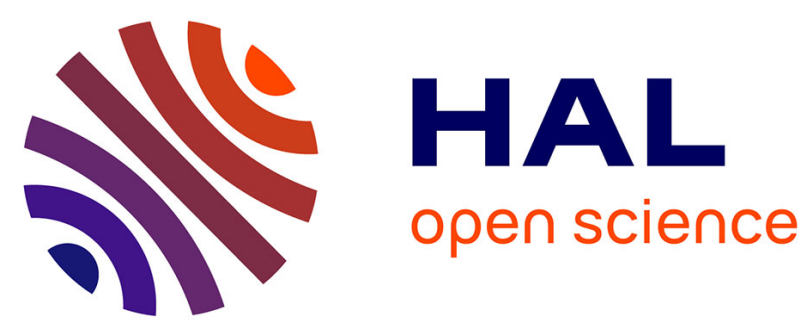

\title{
Energy power spectra measured at an interplanetary shock by the New Horizon's SWAP experiment: 1D full particle simulations versus observations
}

Bertrand Lembège, Zhongwei Yang, Gary P. Zank

\section{To cite this version:}

Bertrand Lembège, Zhongwei Yang, Gary P. Zank. Energy power spectra measured at an interplanetary shock by the New Horizon's SWAP experiment: 1D full particle simulations versus observations. The Astrophysical Journal, 2020, 890 (1), pp.48. 10.3847/1538-4357/ab65c5 . insu-02428121

\section{HAL Id: insu-02428121 \\ https://hal-insu.archives-ouvertes.fr/insu-02428121}

Submitted on 5 Jan 2020

HAL is a multi-disciplinary open access archive for the deposit and dissemination of scientific research documents, whether they are published or not. The documents may come from teaching and research institutions in France or abroad, or from public or private research centers.
L'archive ouverte pluridisciplinaire HAL, est destinée au dépôt et à la diffusion de documents scientifiques de niveau recherche, publiés ou non, émanant des établissements d'enseignement et de recherche français ou étrangers, des laboratoires publics ou privés. 


\title{
Energy power spectra measured at an interplanetary shock by the New Horizon's SWAP experiment: 1D full particle simulations versus observations
}

\author{
Short title : Energy spectra measured at an interplanetary shock
}

Bertrand Lembège* (1), Zhongwei Yang\# $(2,3)$ and Gary P Zank (4, 5)

\begin{abstract}
${ }^{1}$ LATMOS -IPSL-CNRS, Guyancourt 78280, France; * bertrand.lembege@ latmos.ipsl.fr
${ }^{2}$ State Key Laboratory of Space Weather, National Space Science Center, Chinese Academy of Sciences, Beijing 100190, China; \# zwyang@ spaceweather.ac.cn

${ }^{3}$ CAS Key Laboratory of Geospace Environment, Chinese Academy of Sciences, Department of Geophysics and Planetary Science, University of Science and Technology of China, Hefei 230026, China

${ }^{4}$ Center for Space Plasma and Aeronomic Research (CSPAR), University of Alabama in Huntsville, Huntsville, AL 35805, USA;

${ }^{5}$ Department of Space Science, University of Alabama in Huntsville, Huntsville, AL 35899, USA
\end{abstract}

Received 2019 November 1; revised 2019 December 20; accepted 2019 December 23;

\begin{abstract}
One-dimensional particle-in-cell (PIC) simulations are used to analyze the energy spectra measured by the New Horizons' Solar Wind Around Pluto (SWAP) instrument in the upstream region of an interplanetary shock observed at a distance of $\sim 34$ a.u. from the Sun. The use of individual populations simulating the different solar wind ion (SWI) and pickup ion (PUI) populations allow us to clearly identify the contribution of each population to the global energy spectra. The important role of shock front obliquity is stressed in the formation of PUIs streaming back along the magnetic field into the upstream region far from the front. Energy spectra measured by the SWAP experiment are well recovered in the present simulations. A detailed analysis shows that: (1) the highest energy part of the spectrum is formed primarily by both backstreaming PUI- $\mathrm{H}^{+}$and PUI-He${ }^{+}$; (2) the mid-range part of the energy spectrum is composed of both solar wind $\mathrm{SW}-\mathrm{H}^{+}$and $\mathrm{SW}-\mathrm{He}^{2+}$ incoming ions that are superimposed on the PUI- $\mathrm{H}^{+}$population, and (3) the low energy range is composed of incoming PUI- $\mathrm{H}^{+}$. The agreement between experimental and simulation results is improved by using an initially filled-shell distribution for the $\mathrm{PUI}-\mathrm{H}^{+}$population (instead of a zero-thickness shell), since this affects the low energy part of the spectrum strongly. This means that PUI- $\mathrm{H}^{+}$ions have sufficient time to diffuse onto and fill out a shell distribution after their initial pick up in the heliosphere, indicating that the subsequent cooling has an important impact on the global energy spectrum.
\end{abstract}

\section{Introduction}

As the solar wind (SW) expands radially outward from the Sun, slow interstellar neutral atoms flow into the heliosphere and interact with SW ions via charge exchange (Zank 1999, 2015). The newborn ionized interstellar pickup ion immediately starts to gyrate around the interplanetary magnetic field (IMF) and is accelerated by the motional electric field of the SW; hence the term "pickup ions" (PUIs). Typically, depending on the orientation of the IMF 
with respect to the inflowing neutral atoms (primarily $\mathrm{H}$ and a smaller fraction of neutral $\mathrm{He}$ ), the continued ionization of neutral interstellar atoms and their random gyrophase leads to the formation of a ring-beam distribution of PUIs initially. The formed PUI velocity distribution differs from that of the core SWI population, and PUIs have a range of velocities that extends from zero (their initial neutral flow speed) to twice the SWI velocity in the solar wind reference frame depending on the phase of their gyromotion. The ring-beam distribution is unstable and PUIs scatter in both pitch angle and velocity space due to the effects of both pre-existing SW turbulence and waves triggered by the microinstabilities associated with the unstable distribution. The scattering results in the formation of an isotropic shell distribution. As the shell is advected outward from the Sun, the PUIs adiabatically cool, resulting in a filled shell distribution with older PUIs inside the outermost shell as new PUIs are scatter onto the outer shell of the distribution. Indeed, this basic transport process is observed by the New Horizon's SWAP instrument in its measurements of both the basic PUI and SW plasma moment integrated distributions and well described theoretically (Zank et al., 2018). Zhao et al. (2019) have further extended these moment-integrated transport results to the filled shell PUI distribution function itself, including the PUI heat flux induced by the turbulent scattering of PUIs.

The New Horizon's (NH) mission was been launched on January 19, 2006 and completed its Pluto encounter during July 2015. The NH SWAP (Solar Wind Around Pluto) instrument is a top-hat analyzer that detects ions in the energy range $0.035 \sim 7.5 \mathrm{keV} / \mathrm{q}$ (McComas et al., 2008; Elliott et al., 2016). SWAP has made high-resolution measurements of SW ions to at least 41 au from the Sun (McComas et al., 2017) and corresponding high-resolution measurements of the PUI velocity distribution, thanks to its large field of view. SWAP has made direct measurements of interstellar PUI- $\mathrm{H}^{+}$and PUI-He ${ }^{2+}$ components (McComas et al., 2017) co-moving with the SW out from 22 to 38 a.u. Figure 1(a) is an example of the energy spectrum observed by the SWAP experiment at 25.7 a.u. (McComas et al., 2017), where data were accumulated over a 24 hour preiod.

Observations of both PUI-He ${ }^{2+}$ and PUI- $\mathrm{H}^{+}$at an interplanetary (IP) shock in the environment of Pluto at 34 a.u. were described recently by Zirstein et al. (2018). A striking observation made by SWAP was the discovery of a tail in the PUI distribution downstream of the shock for energies above the PUI- $\mathrm{H}^{+}$cut off. Typical measurements are shown in Figure 1(b) (Zirstein et al., 2018). Upstream of the shock front, the SW- $\mathrm{H}^{+}$and the SW-He ${ }^{2+}$ (twice the energy per charge) ions, are relatively cold, and the PUI- $\mathrm{H}^{+}$ions are clearly identified, but there a tail population is also present at energies above the PUI- $\mathrm{H}^{+}$cut-off, which was not included in previous models. On comparing the energy spectra of Figure 1(a) (measured in the solar wind) and those in the upstream region of the IP shock in Figure 1(b), a very strong similarity is seen. Instead of attempting to derive the best fit from previous models that describe the upstream region (Vasyliunas \& Siscoe, 1976), and the DS region (Zank et al.,1996, 2010), in as in Figure 1(b), we divide the entire energy range into three parts: a low (yellow), middle (pink) and high (gray) energy range corresponding respectively to $\mathrm{PUI}-\mathrm{H}^{+}$, the SW ion populations (both $\mathrm{SW}-\mathrm{H}^{+}$and $\mathrm{SW}-\mathrm{He}^{2+}$ ), and both $\mathrm{PUI}-\mathrm{He}^{+}$and $\mathrm{PUI}-\mathrm{H}^{+}$ populations. Three different vertical colored bars identify these ranges respectively. In the present paper, we focus on these energy spectra to address three primary questions: (1) can we retrieve the energy spectrum defined within each energy range from the simulations; (2) what is the contribution of each population to the full energy range, and to each energy range, and finally (3) what is the origin of the high energy ions (above the SW- $\mathrm{He}^{2+}$ energy cut off) in the upstream region of the IP shock? 


\section{Numerical simulation conditions}

To answer the above questions, we use a one-dimensional (1-D) full electromagnetic particle-in-cell (PIC) code named Epoch (Arber et al., 2015), to simulate the interaction of the solar wind with a supercritical, collisionless, interplanetary shock propagating within a quasi-perpendicular angular range. The shock is produced by the injection method as in previous PIC simulations (Lee et al., 2005; Matsukiyo \& Scholer, 2007; Yang et al., 2015; Lembege \& Yang, 2016, 2018). To reproduce self-consistently the affect of PUIs and SW ions, five particle species are introduced within our PIC code: three solar wind populations (electrons, SW-H ${ }^{+}$and $\mathrm{SW}-\mathrm{He}^{2+}$ ions) and two pickup populations (PUI-H ${ }^{+}$and PUI-He ${ }^{+}$). All particles are injected from the left side of the simulation box with an inflow upstream drift speed $V_{i n j}$, and are reflected at the other end (the reflecting wall). The shock front builds up (as incoming/reflected plasmas interact) and moves with a speed $V_{\text {ref }}$ from the right to the left along the $-x$ axis. The initial distribution functions for the SW ions and electrons are Maxwellian. PUIs are distributed on a zero-thickness sphere (unless indicated differently) in velocity space centered at $\mathrm{V}_{\text {inj }}$ with radius $\mathrm{V}_{\text {shell }}$ as in earlier works (Lipatov \& Zank, 1999, Lee et al., 2005; Matsukiyo and Scholer, 2011; Oka et al., 2011; Yang et al., 2012a; Kumar et al., 2018). The spatial resolution $\mathrm{dx}=0.005 \mathrm{~d}_{\mathrm{i}}$, where $\mathrm{d}_{\mathrm{i}}$ is the ion inertial length in the upstream $\left(=\mathrm{c} / \omega_{\mathrm{pi}}\right)$, and the temporal resolution js $\mathrm{dt}=1.5 \mathrm{e}-4 \omega_{\mathrm{ci}}^{-1}$. The units of velocity, spatial length, and time are the Alfven velocity $V_{A}$, the ion inertial length $d_{i}$, and the inverse ion gyrofrequency $\omega_{\mathrm{ci}}{ }^{-1}$, respectively. The initial injection-bulk velocity of the upstream plasma is $V_{\text {inj }}=7 V_{A}$.

The upstream Alfven Mach number of the shock is $M_{A}=\left(V_{i n j}+V_{r e f}\right) / V_{A}$, where the Alfven speed $V_{A}$ is equal to 1 . The ambient magnetic field $B_{0}$ lies in $x-y$ plane, and the shock normal angle $\theta_{\mathrm{Bn}}\left(=55^{\circ}\right)$ is defined between $B_{0}$ and the $-x$ axis. All basic parameters are as follows: Plasma box size length $L_{x}=2100 c / \omega_{p i}$; Mach number $M_{A}=9-11$; light velocity $c=30$, mass ratio $m_{i} / m_{e}=100$, and the electron plasma to cyclotron frequency ratio $\omega_{\mathrm{pe}} / \Omega_{\mathrm{ce}}=3$. The solar wind and pickup plasma parameters are chosen to be as similar as possible to the local plasma conditions in the Pluto environment observed by SWAP (Zirnstein et al., 2018), shown in Table 1. The ion plasma beta is assumed to be $\beta_{\mathrm{i}}=0.04$ (the ratio of the ion kinetic pressure to the magnetic pressure), while the electron plasma beta $\beta_{\mathrm{e}}=0.5$ is chosen as in previous PIC simulations. Initially, 200 particles for each species are used in a cell. The upstream plasma is quasi-neutral, i.e. $n_{e}=n_{i}$, where $n_{i}=n_{S W I}+n_{P U I}=N_{S W I} \times S W I \%+N_{P U I} \times P U I \%$, where $n_{e}, n_{i}, n_{S W I}$ and $n_{P U I}$ are the densities of the electrons (subscript " $e$ "), of the total ions (subscript " $i$ "), of the SW ions (subscript " $S W I$ '), and of the PUIs (subscript "PUI"), respectively. $N_{S W I}, N_{P U I}$, $S W I \%$ and $P U I \%$ are the counts of each ionic species and their relatively weighted percentage in the PIC simulation, respectively. We use the following percentage ratios, SWI-He $e^{2+}$ / $S W I-H^{+}=4 \%$, and $P U I-H^{+} / S W I-H^{+}=4 \%$, which approximate the observed conditions. The percentage of $P U I-\mathrm{He}^{+} / \mathrm{SWI}-\mathrm{H}^{+}=0.8 \%$ is so low that PUI-He ${ }^{+}$can be treated as test particles, which saves computer time.

\section{Simulation results}

\subsection{Affect of backstreaming PUIs on the total energy spectra}

A simple way to observe energetic particles upstream of a shock front is to consider an oblique (quasi-perpendicular) shock, since a certain percentage of the incoming ions interact with the shock front, at which they are reflected and then stream far upstream into the solar 
wind. A numerical parametric analysis has been performed to determine the appropriate conditions (in terms of the Alfven Mach number $M_{A}$ regime and obliquity of the shock front) to produce a noticeable number of backstreaming ions. Note that the energetic ions in the SWAP observed spectra (above the SWI-He ${ }^{2+}$ energy cut-off) correspond to PUI populations (and not to SW ions). In the present results, we mainly focus on a single reference simulation for a shock with $M_{A}=9-11$ and $\theta_{\mathrm{Bn}}=55^{\circ}$. The results are shown in Figure 2. A two-step technique is used to identify the backstreaming ions: first, we separate the incident ions into two groups, those that are reflected and those that are directly transmitted at the shock front, as in previous work (Yang et al., 2012b); second, we keep track of all reflected ions until the end of the simulation. If a reflected ion has a parallel velocity directed upstream and is never advected downstream, the particle will be selected as a backstreaming ion.

The main field components are shown in Figure 2(a). Figures 2(b-f) show electron phase space and ion phase space $(x, v / /)$ plots where the velocity component $v_{/ /}$is calculated with respect to the local $\mathrm{B}$ field. These results clearly show the formation of backstreaming-PUIs for both PUI-H ${ }^{+}$and PUI-He ${ }^{+}$(Figure 2(e,f)). The simulation box $\left(L_{x}=2100 \mathrm{c} / \omega_{p i}\right)$ and the time length of the run $\left(T_{e n d}=150 \Omega_{c i}{ }^{-1}\right.$, where $\Omega_{\mathrm{ci}}$ denotes the proton cyclotron frequency) have been chosen to be long enough to get a well mature shock front and to follow all populations interacting with the shock front, including in particular the heavy ions $\mathrm{He}^{+}$and $\mathrm{He}^{2+}$. In contrast, no backstreaming-SW ions are observed in Figures 2(c, d). The modulation observed in the upstream region of the SW ions is due to a two-stream type instability excited between the incoming/reflected PUIs populations. The reasons for the absence of backstreaming-SW ions, the acceleration mechanism responsible for the formation of backstreaming-PUIs and the precise identification of the two-stream instabilities are beyond the scope of the present paper and will be analyzed in a separate study (see Zank et al., 1996 for a related theoretical analysis).

Figure 3 shows the energy spectra measured within a large sampling box $\left(\Delta x=300 \mathrm{c} / \omega_{p i}\right)$ illustrated by a red rectangle superimposed in the particle phase space $(\mathrm{x}, \mathrm{V} / /)$ of Figure 2 . The sampling box is located at an upstream distance of $20 \mathrm{c} / \omega_{\mathrm{pi}}$ from the ramp. Figure 3(a) shows the global spectrum which can be directly compared to the observations shown in Figure 1(a) (or the observations measured in the upstream region of the IP shock. Figure 1(b)). Figure 3(b) shows the contribution of each of the five populations to the global or total spectrum (where incoming and backstreaming ions are not separated but are all included without distinction). Figure 3(c) shows the contribution of each sub-population to the global distribution (where incoming and backstreaming ions are separated). Red and blue colored curves distinguish $\mathrm{H}^{+}$ and heavy ions $\left(\mathrm{He}^{+}\right.$and $\left.\mathrm{He}^{2+}\right)$ respectively. Good agreement is found between observations and the simulation results, which is clear in terms of the global spectrum and the identification of the three main energy ranges (Figure 3(c) and Figure 1). By distinguishing between the incoming and backstreaming sub-populations, we determine clearly which population contributes to each of the three energy ranges: (1) We find that the low energy range (yellow) is maid up primarily of incoming PUI- $\mathrm{H}^{+}$. (2) The middle energy range (pink) is comprised of SW ions populations that are superimposed on the incoming PUI- $\mathrm{H}^{+}$. (3) The high-energy range (above the SW ion cut off) is more complicated. To clarify the spectral structure of this energy regime, four successive cutoffs can be defined (as energy increases), illustrated by the vertical dashed lines in Figure 3(c). Here, "c1" is the cut-off for the incoming PUI-H $\mathrm{H}^{+}$, "c2" that of the incoming PUI-He ${ }^{+}$, "c3" for the BS-PUI-H", and "c4" for BS-PUI-He'. Note that the cut off "c2" allows us to separate the PUI-He ${ }^{+}$population into two parts, namely the incoming and the backstreaming PUI-He$e^{+}$. Similarly, the cut off "c1" separates the same PUI- $\mathrm{H}^{+}$population into incoming and backstreaming $\mathrm{PUI}-\mathrm{H}^{+}$. This approach allows us to 
determine more simply what population contributes to what part of the spectrum above the SW ion cutoff. There is some interplay and movement of particles from one to another of the four subpopulations within the high-energy range (between "c1" and "c4"), depending on the different plasma conditions (upstream plasma $\beta, M_{A}$ regime, shock obliquity). This can lead to different spectral shapes within the high-energy range. This will be further analyzed in a separate study. Note that the high-energy range is carried by backstreaming ions of both PUI populations only (each having its own cut off) and not backstreaming SW ions.

Further analysis of the simulation and observed spectra reveals some differences. In particular, (1) in the low energy range, the shoulder decreases strongly in Figure 3(a) but smoothly in Figure 1 when approaching very low energy values, and (2) there is a difference in the high-energy range between the observations and the simulation results. These differences are investigated further below.

\subsection{Affect of using a PUI-H+ ${ }^{+}$shell}

The spectra of Figure 3 were obtained using a zero-thickness shell model for both the PUI- $\mathrm{H}^{+}$and PUI-He ${ }^{+}$populations. This however neglects the effects of adiabatic cooling after a PUI was created. Since the low energy range of the total spectrum is determined mainly by the PUI-H ${ }^{+}$(Figure 1), we can anticipate that the adiabatically cooled PUIs may contribute to this part of the spectrum. We therefore replace the zero-thickness shell by a filled-in shell for the PUI- $\mathrm{H}^{+}$population only and perform a separate simulation run. The resulting energy spectra are shown in Figures 4(a,c) at time $t=0$ (used as a reference time) and in Figures 4(b, d) at time $t=150 \Omega_{c i} i^{-1}$, respectively. The conditions under which the spectra are derived are identical for both simulations i.e. the sampling box (width of $\Delta x=300 \mathrm{c} / \omega_{p i}$ ) is located at a distance of $20 \mathrm{c} / \omega_{p i}$ from the shock ramp (see Figure 2 for a reference). The spectra are presented using the same scales for a direct quantitative comparison. The main results for the filled-shell case at $t=150 \Omega_{c i}{ }^{-1}$ are summarized as follows:

(1) Very good agreement between simulation and observation is now found for the low energy regime (yellow area in Figure 4(d)) (see Figure 1). This is easily understood by noting that in the solar wind PUI- $\mathrm{H}^{+}$has enough time to scatter in pitch angle and velocity space (due to the intrinsic turbulence of the expanding solar wind - see Zank et al., 2018) to cool adiabatically and fill-in the shell. This result also shows that the generation of upstream waves by a two-stream type instability between incident/backstreaming populations (Figures 2(b-f)) is insufficient to strongly scatter and diffuse the PUIs and to fill-in the initial zero-thickness shell.

(2) A main peak now forms in the spectral region corresponding to incident PUI- $\mathrm{H}^{+}$ (being superimposed on $\mathrm{SW}-\mathrm{H}^{+}$ions) in contrast to observational results that show such a maximum is shifted to a higher energy (near the location of SW-He ${ }^{2+}$ ions). This is in contrast with the zero-thickness shell case that exhibited a relatively flat sub-spectrum in the middle energy range. These differences require further investigation, which is beyond the scope of the present paper.

(3) The distinction between incoming and backstreaming-PUI- $\mathrm{H}^{+}$sub-spectra is much clearer in the high-energy range, and a similar improvement distinguishing between backstreaming PUI- $\mathrm{H}^{+}$and backstreaming PUI-He ${ }^{+}$ions results in a better agreement between observations and simulation results.

(4) The smaller cut-off for backstreaming-PUI-H ${ }^{+}$shows that the high-energy range of backstreaming-PUIs (within the gray area in Figure 4(d)) is shrunk for the filled-in shell 
compared with the zero thickness shell case, i.e. the energization is more limited;

(5) A surprising result is the impact that a filled-shell PUI- $\mathrm{H}^{+}$distribution has on both SW ion populations. Both exhibit a cooler distribution at $t=150 \Omega_{c i}^{-1}$ (the width of each distribution decreases for the filled-in shell case), compared to the zero-thickness shell case.

(6) The cut-off "c4" of the highest energy ions (backstreaming-PUI-He ${ }^{+}$) is unaffected by a filled PUI-H ${ }^{+}$shell distribution. These combined effects lead to a relatively flat high-energy tail in the highest energy range of the spectra, which is consistent with previous observations (McComas et al., 2017; Zirstein et al, 2018).

Moreover, simulations have been also performed in the case where both PUI populations $\left(\mathrm{H}+\right.$ and $\left.\mathrm{He}^{+}\right)$are initially described as filled-in shells. A few differences can be noticed in the corresponding spectra shown Figures $4(\mathrm{e}, \mathrm{f})$ with respect to the zero-thickness shell case (Figures $4(a, b)$ ): (i) as expected, the shoulder in the high energy range of PUI-He ${ }^{+}$ is much smoother at $\mathrm{t}=0$; (ii) the percentage of backstreaming PUI- $\mathrm{He}^{+}$is much lower (Figure 4f); (iii) the high energy range covered by the backstreaming-PUI (grey area covered by both PUIs- $\mathrm{H}^{+}$and PUIs-He ${ }^{+}$) is almost not affected; (iv) the transition between middle- (pink area) and high-energy (gray area) range is smoother; (v) in the middle energy range, the sub-spectrum of incoming PUI- ${ }^{+}$presents a peak corresponding to the peak of SWI- $\mathrm{H}^{+}$ population; similar result is also observed between the peaks of incoming $\mathrm{PUI}-\mathrm{H}^{+}$and SWI-He ${ }^{2+}$.

In summary, by considering a filled-in rather than a zero-thickness shell for PUI-H ${ }^{+}$, we find that the spectral energy distribution changes quite extensively, especially in the contributions of the different populations within the global or total energy spectrum.

\subsection{Impact of shock front obliquity}

A comparison between the phase space of the different ion populations (Figure 2) and the associated energy spectra (Figure 3) shows that the reflected ions backstreaming far upstream from the front (selected in the sampling area shown in Figure 2) are energetically important and are a key component of the highest energy spectral regime. This implies that shock front obliquity has a significant impact in determining the energetic part of the spectrum. To properly evaluate the importance of shock obliquity to the high-energy part of the spectrum, we undertook a separate simulation for a strictly $90^{\circ}$ IP shock. All other plasma and shock conditions remain unchanged; in particular, zero-thickness shells were used for PUIs as for the reference run (Figure 2). For the $90^{\circ}$ case, reflected ions can only accumulate over a short distance from the ramp, i.e., in the foot of the perpendicular shock, since under the effect of the magnetic field the PUIs are forced to gyrate and return to the front. Consequently, no backstreaming ions can escape upstream from the ramp. Corresponding spectra obtained for $55^{\circ}$ and $90^{\circ}$ shocks are compared and shown in Figure 5. The main features can be summarized as follows:

(1) As expected, the overall range of particle energies for the $55^{\circ}$ shock is much larger than for the $90^{\circ}$ shock, which confirms that the high-energy part of the spectrum is due primarily to both backstreaming-PUI $\mathrm{H}^{+}$and $\mathrm{He}^{2+}$.

(2) The spectrum of each PUI population exhibits a plateau (corresponding to the zero-thickness shell of PUIs) with abrupt limits in contrast to the smooth shoulders present for $55^{\circ}$ shock case.

(3) The energy range of $\mathrm{SW}-\mathrm{H}^{+}$and of $\mathrm{SW}-\mathrm{He}^{2+}$ ions is much smaller for the $90^{\circ}$ shock than for the $55^{\circ}$ case. This is because, for the $55^{\circ}$ case, the backstreaming PUIs 
/incoming populations interact, leading to the development of a two-stream type instability. The instability affects all particle populations incident on the shock, as illustrated by the modulations in phase space of not only PUIs but also SW electrons and of SW ions (Figure 2). Hence, both PUI and SW ion populations experience some heating due to the instabilities generated by the backstreaming-PUI populations. For the $90^{\circ}$ shock, the Maxwellian SW ion distribution corresponds to the pristine solar wind only and is unaffected far upstream of the shock front since there are no instabilities triggered by backstreaming ions.

\section{Discussion and conclusions}

The work presented here was stimulated by the first direct observations of the mediation and preferential heating of non-thermal PUIs rather than thermal SW ions upstream of an interplanetary shock in the environment of Pluto. To our knowledge, this study is one of the first attempt to understand these recent measurements using self-consistent plasma simulations. Although the simulations presented herein are one-dimensional, they can reproduce the most prominent features of the observed ion energy spectra. These observations were made by the SWAP experiment on the New Horizons mission at a distance of 34 a.u. from the Sun (Zirnstein et al., 2018). At this distance, PUI- $\mathrm{H}^{+}$is only a few percent of the total proton density but contains most of the internal particle pressure (see Zank et al., 2018). Moreover, the observed energy spectra are very similar to those observed at a distance of 22-38 au by SWAP (McComas et al., 2017).

The results presented here show (i) how different parts of the total or global spectrum observed upstream of an IP shock can be understood in terms of the contributions from the different populations (essentially forming sub-spectra), and (ii) how certain parameters influence these different "subspectra," allowing us to refine the comparison between observations and simulation results. The simulation results reflect a self-consistent approach and account for the time/spatial scales of all populations. Results presented herein describe the upstream region of an interplanetary shock only and will be extended to the downstream region in a subsequent analysis. Our primary results can be summarized as follows:

a) Shock obliquity plays a key role in the formation of energetic PUIs streaming back far upstream from the shock ramp. No backstreaming SW ions are observed.

b) The "strategy" used herein differs from previous analyses. Instead of fitting different charged particle populations with modeled distribution functions (e.g., Zank et al., 2010), we decomposed the total energy spectrum into three energy ranges, i.e., low-, middleand high-energy ranges. The three energy ranges correspond respectively to (1) incident PUI- $\mathrm{H}^{+}$mainly; (2) both SW- $\mathrm{H}^{+}$and $\mathrm{SW}-\mathrm{He}^{2+}$ ions that are superimposed on incident PUI-H ${ }^{+}$ population, and (3) both backstreaming PUI- $\mathrm{H}^{+}$and backstreaming PUI-He ${ }^{+}$. We emphasize that the backstreaming-PUI populations account quite well for the highest energy part of spectrum above the incoming PUI-H ${ }^{+}$cut-off. Three other cut-offs can be identified at higher energies, (i) one for incoming PUI-He${ }^{+}$; (ii) one for backstreaming PUIs- $\mathrm{H}^{+}$, and (iii) one for backstreaming PUI-He ${ }^{+}$. This approach allowed us to determine which part of the spectra (and the corresponding sub-population) is primarily sensitive to various upstream plasma conditions.

c) When a filled-shell distribution (rather than a zero-thickness shell) is used to describe both the initial PUI- $\mathrm{H}^{+}$and PUI-He ${ }^{+}$populations, a better agreement is observed in the low- and high-energy ranges between observational and simulations results. This is important in that it confirms that PUIs do have sufficient time to diffuse in velocity space after the initial pick up in the supersonic solar wind. However, there are differences between 
observations and simulations in the middle energy range, which requires further investigation.

Observational results presented by McComas et al. (2017) and Zirnstein et al., (2018) exhibit different shapes in the global energy spectra, in particular on the "sharpness" of the shoulders that are present in the high-energy regime that is formed primarily by $\mathrm{PUI}-\mathrm{H}^{+}$and PUI- $\mathrm{He}^{+}$. The results presented here are preliminary and focused on one typical observed spectrum, and further parametric analyses will be explored.

\section{Acknowledgments}

We acknowledge the French Centre National d'Etudes Spatiales (CNES) for its support under APR- W-EEXP/10-01-01-05 et APR-Z-ETP-E-0010/01-01-05. Much of the numerical simulations were done on the IDRIS computer center located at Orsay (near Paris), which we thank for its support (DARI project A0050400295). A part of the computations were performed by Numerical Forecast Modeling R\&D and VR System of State Key Laboratory of Space Weather. The research is also partially supported by NFSC under Grant No. 41574140, the Open Research Program Key laboratory for the Geospace Environment CAS, Youth Innovation Promotion Association Funding No. 2017188, Beijing Municipal Natural Science Foundation No. 1192018, Beijing Excellent Talent Training Project Funding No. 2017000097607G049, and the Specialized Research Fund for State Key Laboratories of China. GPZ acknowledges the partial support of the NSF EPSCoR RII-Track-1 Cooperative Agreement OIA-1655280, an NSF/DOE Partnership in Basic Plasma Science and Engineering via NSF grant PHY-1707247, and NASA grant/ subaward 0000167 (NNX17AB04G). One of the authors (ZY) would like to thank LATMOS for its nice hospitality during his stay.

\section{References}

Arber, T. D., Bennett, T. D., Brady, C. S., et al. 2015, PPCF, 57, 113001

Elliott, H. A., McComas, D. J., Valek, P., Nicolaou, G., Weidner, S., \& Livadiotis, G. 2016, ApJS, 223, 19

Lee, R. E., Chapman, S. C., \& Dendy, R. O. 2005, AG, 23, 643

Matsukiyo, S., Scholer, M., \& Burgess, D. 2007, AG, 25, 283

Matsukiyo, S., \& Scholer, M. 2011, JGR, 116, A08106

McComas, D., Allegrini, F., Bagenal, F., et al. 2008, SSRv, 140, 261

McComas, D. J., Zirnstein, E. J., Bzowski, M., et al. 2017, ApJS, 233, 8

Lembege, B., \& Yang, Z. W. 2016, ApJ, 827, 73

Lembege, B., \& Yang, Z. W. 2018, ApJ, 860, 84

Lipatov, A.S. \& Zank, G.P., 1999, PRL, 84, 3609-3612

Kumar, R., Zirnstein, E. J., \& Spikovsky, A. 2018, ApJ, 860, 156

Oka, M., Zank, G. P., Burrows, R. H., \& Shinohara, I. 2011, AIP Conf. Proc., 1366, 53

Vasyliunas, V. M., \& Siscoe, G. L. 1976, JGR, 81, 1247

Yang, Z. W., Han, D. S., Yang, H. G., et al. 2012a, ASS, 341, 241 
Yang, Z. W., Lembege, B., \& Lu, Q. M. 2012b, JGR, 117, A07222

Yang, Z. W., Liu, Y. D., Richardson, J. D., et al. 2015, ApJ, 809, 28

Zank, G. P., Pauls, H. L., Cairns I. H., \& Webb, G. M., 1996, JGR, 101, 457

Zank, G. P., Heerikhuisen, J., Pogorelov, N. V., Burrows, R., \& McComas, D. 2010, ApJ, 708, 1092

Zank, G. P. 1999, SSRv, 89, 413

Zank, G. P. 2015, ARA\&A, 53, 449

Zank, G.P., Adhikari, L., Zhao, L.-L., et al., 2018 ApJ, 869:23, doi/10.3847/1538-4357/aaebfe

Zhao, L.-L., Zank, G.P., \& Adhikari, L., 879:32, doi/10.3847/1538-4357/ab2381

Zirnstein, E.J., McComas, D. J., Kumar, R., et al. 2018, PvRvL, 121, 075102

Figures and Table captions

(a) McComas et al., 2017

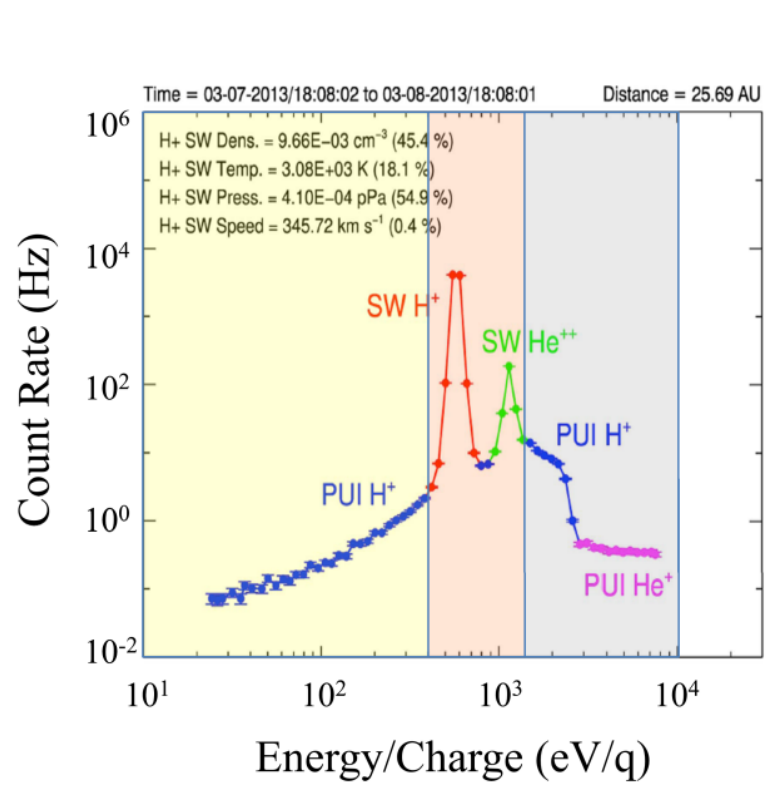

(b) Zirnstein et al., 2018

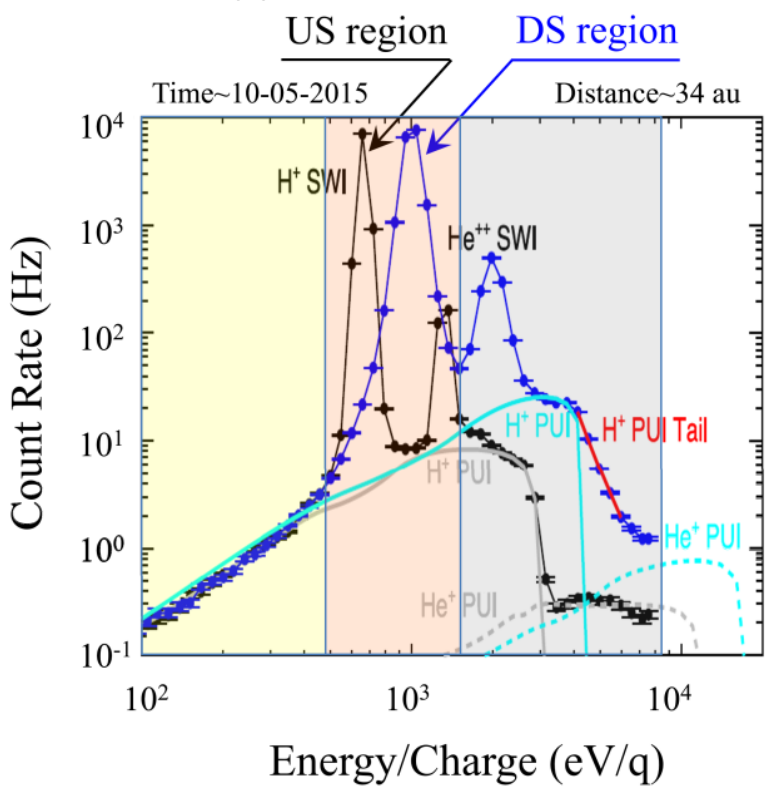

Figure 1. Panel (a) shows a typical example of a SWAP energy spectrum measured at 25.7 a.u., color coded with the primary source of the counts: solar wind ions (SWIs) and interstellar pickup ions (PUIs). Observations were collected over one day and provide highly statistically significant measurements (from McComas and al., 2017). Three energy ranges are identified in the spectrum by the vertical colored areas (see the corresponding text). Panel (b) shows another observation collected by the SWAP instrument before (black) and after (blue) the crossing of an interplanetary shock by New Horizons that was measured near Pluto at 34 a.u. Fits to the PUI-H ${ }^{+}$before (gray) and after (blue) the shock front are shown respectively based on the models of Vasyliunas \& Siscoe, (1976), and Zank et al. $(1996,2010)$ (from Zirnstein and al., 2018). The acronyms "US" and "DS" refer to upstream and downstream regions of the shock respectively. 


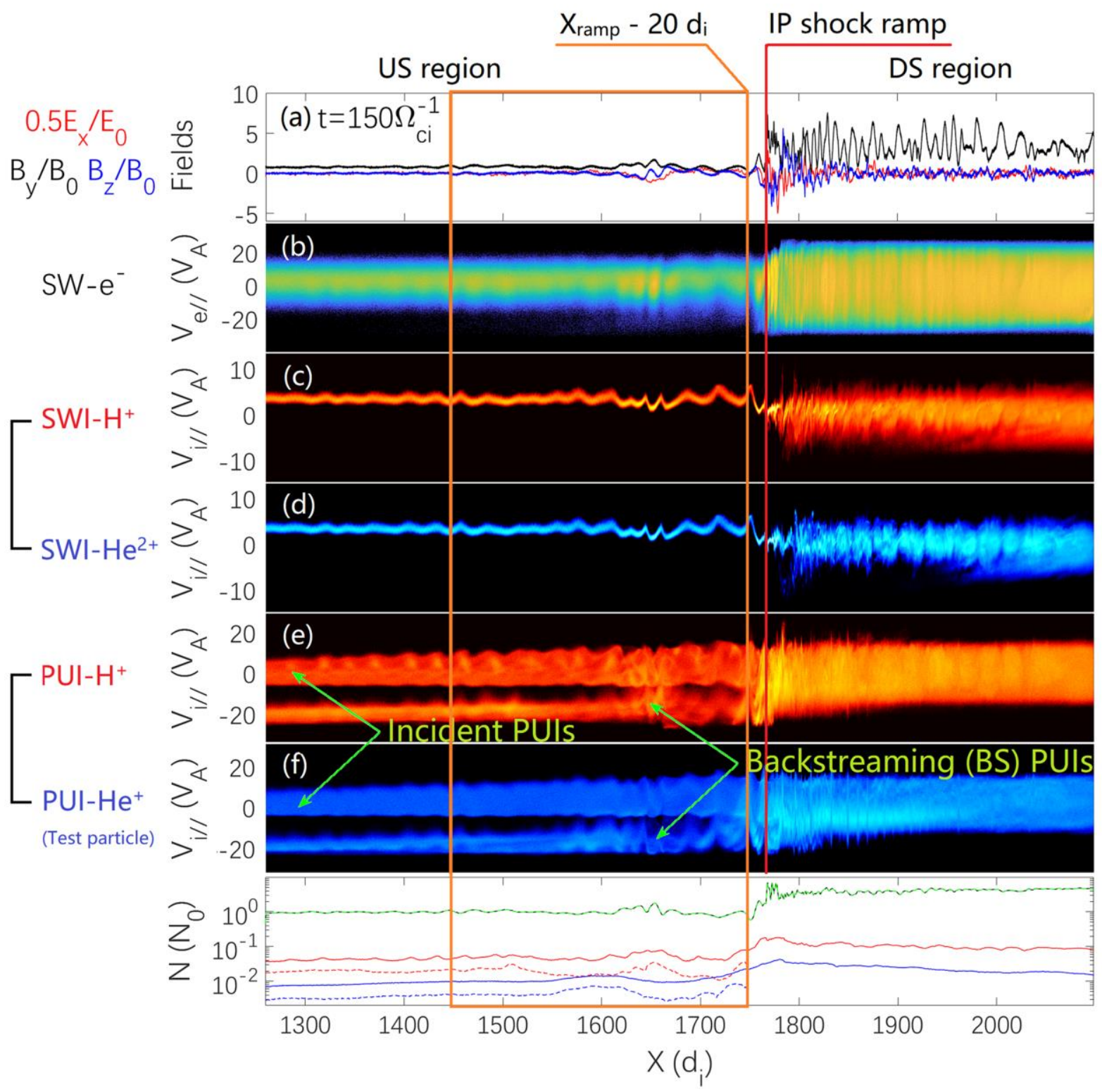

Figure 2. The top plot (a) shows magnetic field profiles $B_{t y}$ (black) and $B_{t z}$ (blue), and the electric field component $E_{x}$ (red) at a late time $t=150 \Omega_{c i}{ }^{-1}$ of the simulation. The plots below show the phase space $(x, v / /)$ for the different populations: (b) electrons; (c) SW-H ${ }^{+}$ ions; (d) SW-He ${ }^{2+}$ ions; (e) PUI-H+, and (f) PUI-He ${ }^{+}$; the parallel velocity component ( $v / /$ ) is defined with respect to the local magnetic field. $(\mathrm{g})$ The total density profiles of PUI-H ${ }^{+}$and PUI-He ${ }^{+}$ions are indicated by red and blue solid curves, respectively. Their corresponding BS parts are represented by dashed curves. The vertical dashed line identifies the ramp location in the shock front. The large colored rectangle $\left(\Delta x=300 \mathrm{c} / \omega_{p i}\right)$ is located at a distance of $20 \mathrm{c} / \omega_{\mathrm{pi}}$ from the ramp and identifies the upstream sampling $x$-range where the energy spectra are calculated (to see next figures). 
(a) Total envelop

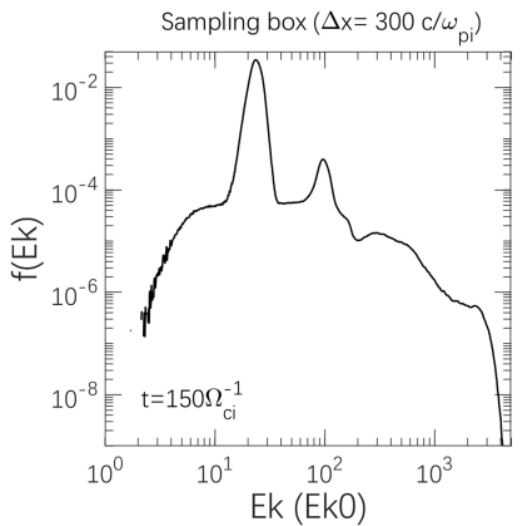

(b) Sub-envelops

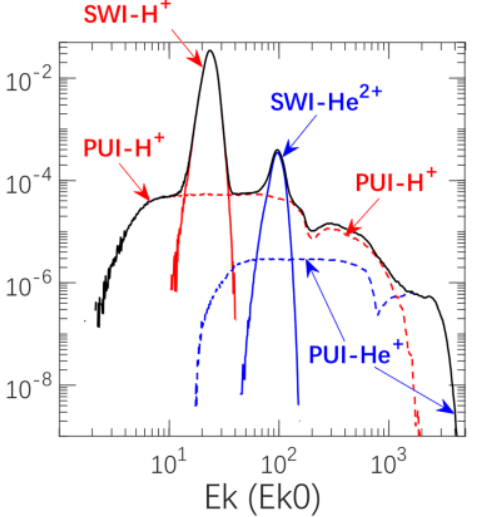

(c) Separated incoming and BS ions

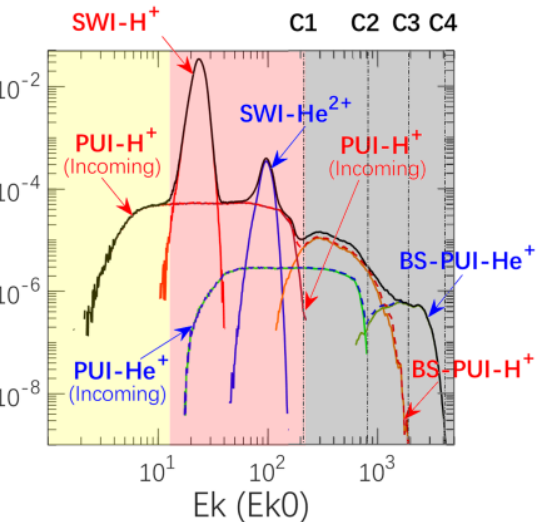

Figure 3. (a) Distribution for the total energy spectrum derived within the sampling range $\Delta x=300 \mathrm{c} / \omega_{p i}$ at a late time $t=150 \Omega_{c i}{ }^{-1}$ of the simulation (to see Figure 2); (b) the same result but now identifying the sub-spectra corresponding to each of the four different ion populations (incoming and backstreaming PUIs are not yet separated); (c) the same results but where now individual energy spectra are identified for each of the six populations (4 incoming ion and 2 backstreaming ion populations, and the incident and backstreaming PUI contributions are now separated). The red and blue colored curves are used for $\mathrm{H}^{+}$and heavy ions $\left(\mathrm{He}^{+}\right.$and $\left.\mathrm{He}^{2+}\right)$ respectively.
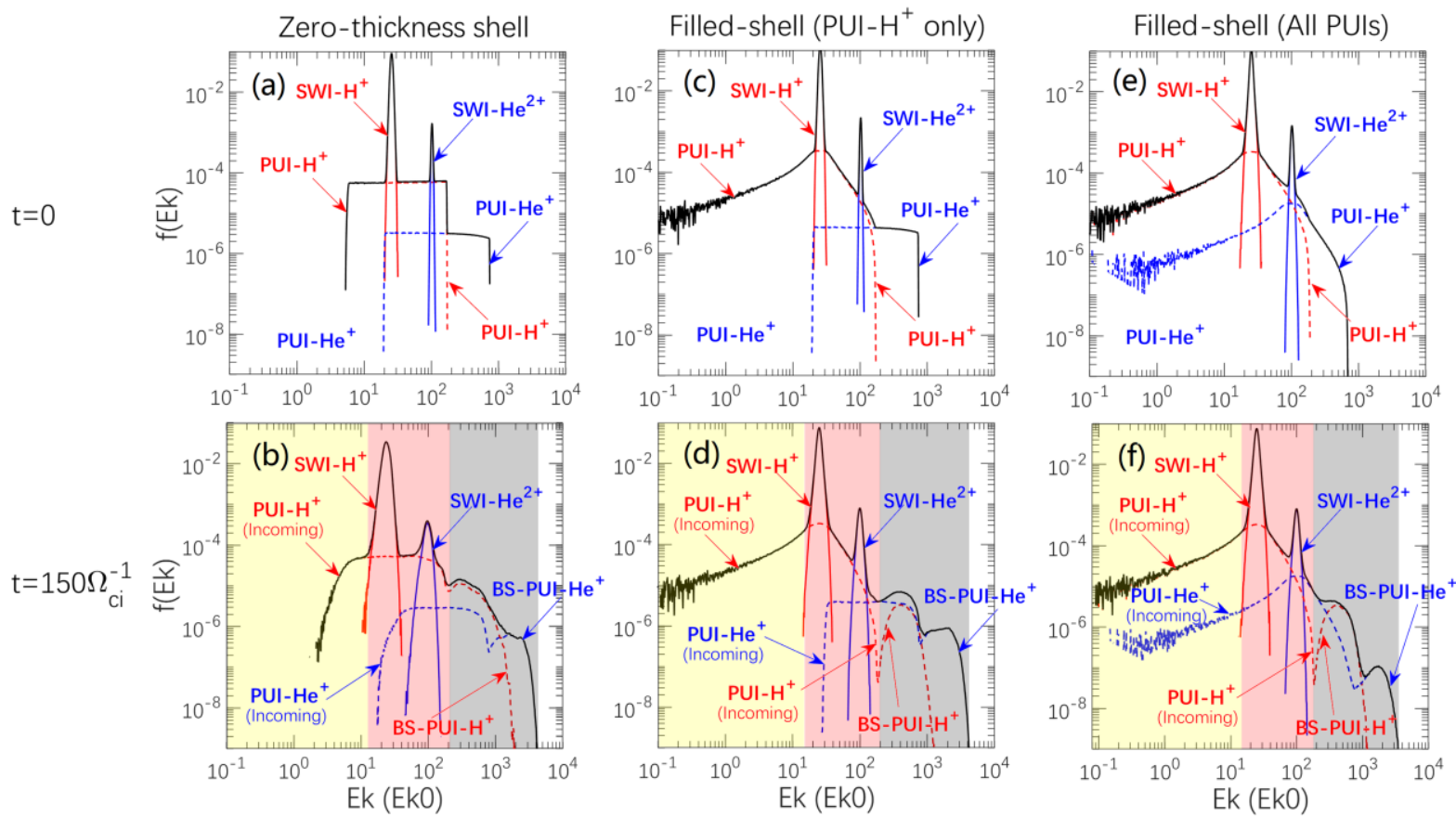

Figure 4. Energy spectrum derived from an initially zero-thickness shell used for both PUI distributions at (a) $t=0$, and (b) at the late time $t=150 \Omega_{c i}{ }^{-1}$ of the simulation. Corresponding spectra are shown as an initially filled-shell distribution is used for PUI-H ${ }^{+}$ distribution only (plots (c) and (d)), and for both PUI-H ${ }^{+}$and PUI-He ${ }^{+}$distributions (plots (e) and (f)). The red and blue colored curves denote $\mathrm{H}^{+}$and heavy ions $\left(\mathrm{He}^{+}\right.$and $\left.\mathrm{He}^{2+}\right)$ respectively; black curve is used for the global spectrum. The same scales are used for all spectra. 

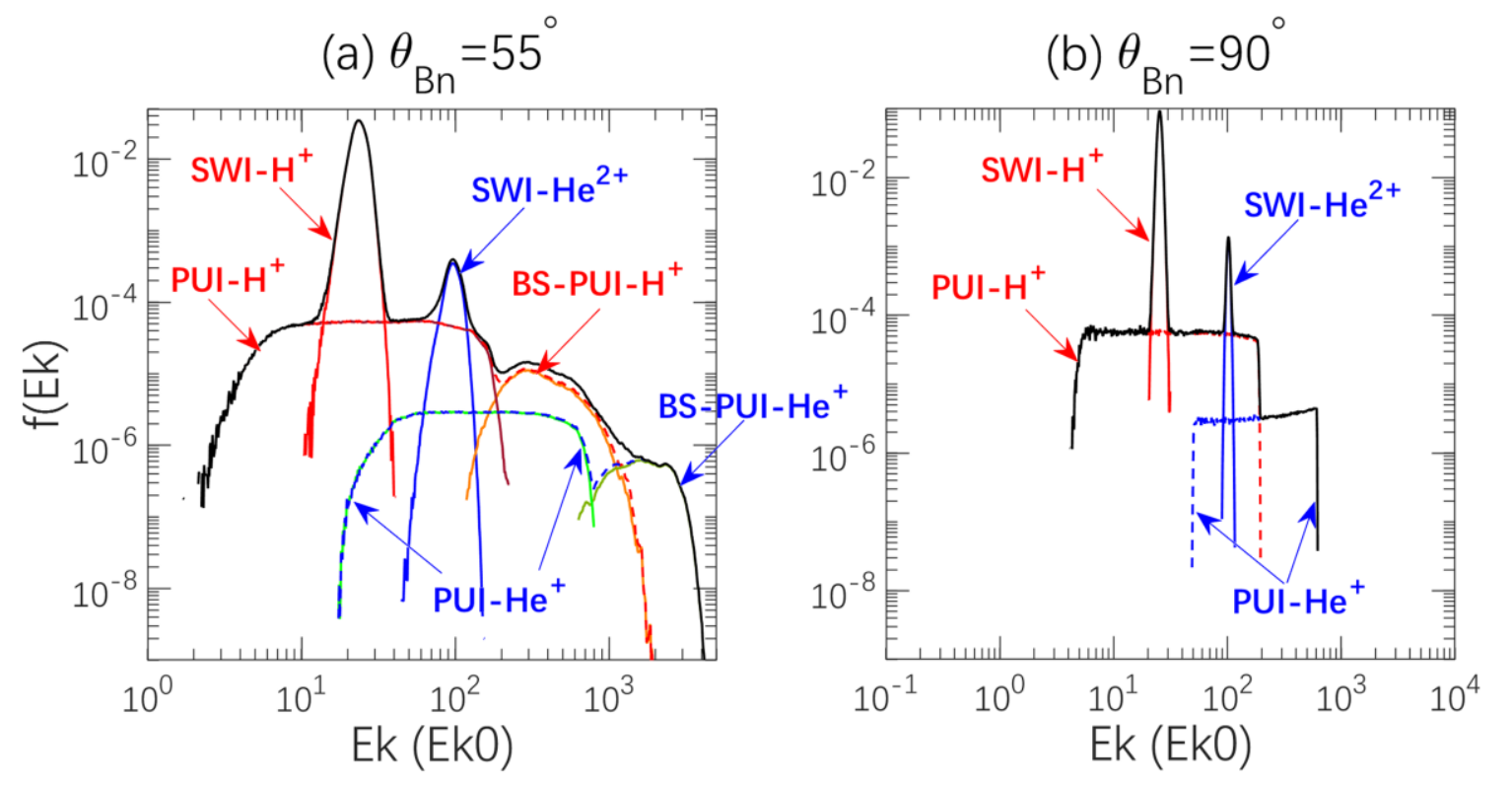

Figure 5. Energy spectra determined upstream of the interplanetary shock with obliquity (a) $\theta_{\mathrm{Bn}}=55^{\circ}$, and (b) $90^{\circ}$ at the same late time $\mathrm{t}=150 \Omega_{\mathrm{ci}}{ }^{-1}$ for each simulation. Spectra are derived within the sampling $x$-range $\Delta x=300 \mathrm{c} / \omega_{p i}$ located at $20 \mathrm{c} / \omega_{\mathrm{pi}}$ from the ramp for (a), and within a restricted sampling $x$-range $\Delta x=15 c / \omega_{p i}$ (since the area of reflected-gyrating ions is quite restricted) located at $15 \mathrm{c} / \omega_{\mathrm{pi}}$ from the ramp for (b) (see text for further discussion). Red and blue colored curves denote $\mathrm{H}^{+}$and heavy ions $\left(\mathrm{He}^{+}\right.$and $\mathrm{He}^{2+}$ ) respectively; black curve is used for the global spectrum. The same scales are used for both spectra.

Table 1. Values of upstream plasma parameters used in the simulations

\begin{tabular}{|l|l|l|l|l|}
\hline Parameter & Description & Electrons & SWIs & PUIs \\
\hline$V_{\text {thxyz }}$ & Thermal velocity & 5.0 & 0.14 & \\
\hline$\lambda_{\mathrm{D}}$ & Debye length & 0.017 & 0.0047 & Shell \\
\hline$\rho_{\mathrm{c}}$ & Gyro-radius & 0.05 & 0.14 & distribution \\
\hline $\mathrm{c} / \omega_{\mathrm{p}}$ & Inertial length & 0.1 & 1 & \\
\hline$\omega_{\mathrm{c}}$ & Gyro-frequency & 100 & 1 & 30 \\
\hline$\omega_{\mathrm{p}}$ & Plasma frequency & 300 & 1 & \\
\hline$\tau_{\mathrm{c}}$ & Gyro-period & 0.01 & 0.04 & \\
\hline$\beta$ & Plasma beta & 0.5 & & \\
\hline
\end{tabular}

